\title{
The Design and Simulation of High Impedance Surface in Airborne Telemetry Antenna
}

\author{
Yan-Lian ZHANG ${ }^{1}$, Hai-Bo GONG ${ }^{1, a}$ and Qian XU1 \\ ${ }^{1}$ Flight test establishment of China, Yanliang District, Xi'an, Shaanxi Province, China,
}

\begin{abstract}
Aiming to the problems that excessive reduction of airborne telemetry antenna's size will cause a sharp deterioration of the antenna's bandwidth and gain, this paper proposed that using a high-impedance surface in the airborne telemetry antenna. As the size and bandwidth of the high-impedance surface is far from being able to meet the application requirements, this paper designed a new high-impedance surface which can be applied to the telemetry transmitter antenna, and reduced the profile size of the telemetry antenna and increased the gain of the antenna at the same time. In addition, based on the equivalent circuit model, we have resolved the contradictory situation of small size and wide band gap in the high-impedance surface design. The results show that the low profile, high gain and wide bandwidth of the telemetry antenna can be achieved by using the new high-impedance surface. The antenna structure and design ideas presented in this paper can significantly improve the performance of airborne telemetry antenna.
\end{abstract}

\section{Introduction}

Real-time data processing and monitoring are often used in flight test. With the increasing number of high-risk item in flight test, real-time remote monitoring is becoming more and more important [1]. The telemetry transmit antenna used in the flight test is a microstrip antenna that adheres a thin metal conductor to a dielectric substrate. With the continuous improvement of aircraft performance requirements, airborne telemetry antenna in high-altitude and high-speed environment has a good aerodynamic shape and excellent performance has become a hot research area [2]. Conventional airborne telemetry antenna size will lead to over-reduction of other antenna performance of the rapid deterioration, in which the bandwidth and gain is particularly evident, so compromise between the various parameters and. Aiming at the problem, the parameters and performance of the telemetry antenna can not be taken into account, we have introduced the high resistance surface structure in the airborne telemetry antenna. The high impedance surface can exhibit high surface impedance in a certain frequency band, can cut off the surface wave propagation, at the same time for the incident plane electromagnetic wave has the same phase reflection characteristics, can replace the metal reflective surface as the antenna ground plate, thereby reducing the antenna profile [3].

The current low-impedance surface can be achieved, but the size and bandwidth is far from meeting the requirements of the application. This paper analyzes the miniaturization and wide bandgap design of the high resistance surface. The new high impedance surface structure can be

\footnotetext{
${ }^{a}$ Corresponding author: 13324521609@163.com
} 
successfully applied to the aerial telemetry antenna, which can realize the low profile, high gain and wide frequency band design of the telemetry antenna. Simulation results using ANSOFT HFSS show that the new structure can make the telemetry antenna have a smaller profile size and better integration of antenna and fuselage without loss of bandwidth, and high antenna gain.

\section{Airborne telemetry antenna and theoretical model of high resistance surface}

In the airborne telemetry system, the telemetry transmitting antenna is a device for radiating electromagnetic wave. It is used to transmit the comprehensive information obtained from the airborne test to the remote telemetry receiving station, to realize the ground real-time data processing and real-time monitoring of the aircraft flight test result. The telemetry transmitting antenna used in aircraft flight test is microstrip antenna which sticks the thin metal conductor on the dielectric substrate. The microstrip patch antenna shown in Fig. 1 is the most common aerial telemetry antenna, layer or multi-layer dielectric layer and covering the upper and lower sides of the radiation element and the metal grounding plate.

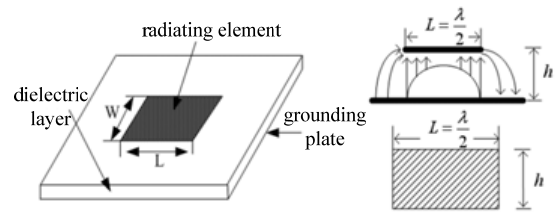

Figure 1. Airborne telemetry antenna and radiation field

A high-impedance electromagnetic surface is a electromagnetic band gap (EBG) structure consisting of a metal and a dielectric material. It is a resonant electromagnetic bandgap structure. The high-impedance electromagnetic surface is a textured surface consisting of a set of metal patches periodically arranged in a two-dimensional grid pattern on the dielectric substrate, and connected to the metal ground plane of the dielectric substrate through a vertical conductive via each metal patch [4]. It is not only the same as other types of electromagnetic bandgap structure with the role of suppression of surface waves, but also in a certain frequency range to achieve the same phase reflection, instead of metal reflective surface as the antenna ground plate, thereby reducing the antenna profile. Figure 2 is the surface structure of the most common high-resistance, square patch in the center with the ground through the hole, the square array arranged.

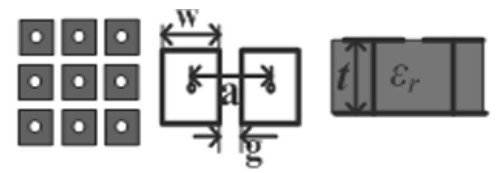

Figure 2. Top view, unit structure and side view

The cell size of the high resistance surface is much smaller than the operating wavelength, so its electromagnetic characteristics can be described by the lumped circuit components. Their characteristics are the same as the parallel LC resonant circuit, the capacitance between the adjacent metal units, the inductance of the conductive path. The equivalent circuit is shown in Figure

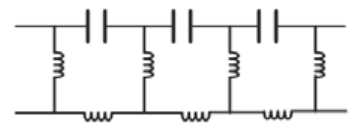

Figure 3. Equivalent circuit of High impedance surface 
According to the equivalent surface impedance model, the resonant frequency of the high-resistance surface cell (the stopband center frequency of the high-resistance surface) and the bandwidth are:

$$
\begin{aligned}
& \varpi_{0}=1 / \sqrt{L C}, \Delta \varpi / \varpi_{0}=\sqrt{L / C} / \sqrt{\mu_{0} / \varepsilon_{0}} \\
& L=\mu_{\mathrm{r}} \mu_{0} \mathrm{t}, \quad C=\frac{\eta\left(\varepsilon_{0}+\varepsilon_{1}\right)}{\pi} \cosh ^{-1}\left(\frac{a}{g}\right)
\end{aligned}
$$

Where $\mu_{0}$ is the permeability in vacuum, $\mu_{\mathrm{r}}$ is the dielectric material relative permeability, $t$ is the thickness of the dielectric substrate, $\varepsilon_{0}$ is the dielectric constant in vacuum, $\varepsilon_{1}$ is the substrate dielectric constant, $w$ is the unit side length, $a$ is the adjacent distance between the holes $g$ is the gap width between the cells.

\section{Compact design of high resistance surface}

In order to improve the performance of the antenna and reduce the backward radiation, microstrip antenna generally placed under the antenna metal reflective surface. At this point, the metal reflective surface and the antenna need to maintain a quarter-wavelength height, profile size is relatively large. For the incident plane electromagnetic waves, high resistance surface in a certain frequency range has the same phase reflection characteristics, and no metal surface of the $180^{\circ}$ phase difference, without a quarter-wavelength profile thickness, so the antenna and high resistance surface has great advantage and potential in realizing the low profile antenna with its great value of electromagnetic property, and it has a very high research value.

In practical application process, the high resistance surface structure must have sufficient number of units, or the electromagnetic properties are not obvious [5]. If the traditional electromagnetic surface structure shown in Fig 2 are used in the airborne telemetry antenna design, the size of $90 \mathrm{~mm}$ $\times 75 \mathrm{~mm}$ area, the number of units can accommodate no more than four, then the high-resistance surface of the electromagnetic properties simply can not be realized. Therefore, in order to integrate the high resistance surface structure into the design of airborne telemetry antenna, the high resistance surface needs to realize the miniaturization design.

By the high resistance surface equivalent circuit model, reducing the bandgap frequency can be realized by increasing the equivalent inductance $\mathrm{L}$ and capacitance $\mathrm{C}$. The equivalent inductance is determined by the material of the high resistance surface, and the inductance is determined when the preparation material is determined. The factors that affect the capacitance are a lot, you can change the surface of the high-impedance surface of the structure of the cycle to change the amount of capacitance [6]. Thus we designed the L seam, F seam embedded structure, as shown in Figure 4 and Figure 5.

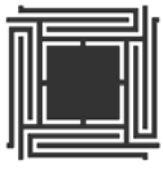

Figure 4. LSeam structure

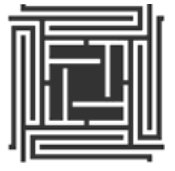

Figure 5. F Seam structure

At present, the realization of compact high impedance surface design is mainly based on the equivalent capacitance to improve the structure to achieve, for example, three-tier structure, interactive embedding, three-tier structure of the implementation process is complex, technical requirements and costs are high, the practical application by restrictions. Compared with the traditional high-resistance surface structure shown in Figure 2, the inter-embedded structure can form a stronger coupling capacitor [7], reducing nearly $60 \%$ of the unit size. However, over a limited 
number of iterations, a limited patch area can not be fully utilized and the cell size can not be further reduced. It is found that the metal slit can make the path of the surface current flow longer and make the equivalent electric dimension of the patch larger, which can enhance the coupling capacitance between the cells. Therefore, the design of the slit structure and the embedded structure are the same, the combination of the two slotted embedded structure can be more effective use of patch area, thereby more effectively reduce the size of the unit. We designed the F-slot embedded structure which can reduce the cell size of more than $70 \%$, to achieve high-resistance surface structure of the small size of the design effect is significant.

As shown in Figure. 6, the digital phase-change curve of the reflection phase corresponds to the L-shaped gap from the center of the patch to the close-to-close patch. The simulation of the slit-embedded structure shows that the width of the slit is constant. Slice edge of the process of change. Therefore, in order to effectively reduce the cell size, the L-shaped slot can be opened at the outermost side of the patch. F-type gap from the L-type gap and straight-line gap composition. To maintain the L-shaped gap in the outside of the patch, we adjust the F-shaped slot straight seam position, the reflection phase of the impact shown in Figure 7. The closer to the center of the patch, the greater the effect on the resonant frequency. F-type gap can adjust the edge of the patch and the inside surface current, to minimize the size of the unit. In addition to the location of the gap, the gap length of the resonant frequency of the greatest impact, the wider the gap, the longer the lower the resonant frequency.
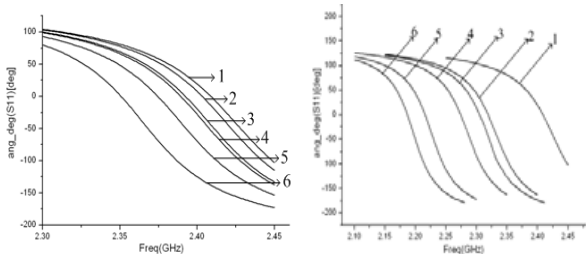

Figure 6. Change of L Slit phase Figure7 change of F Slit phase

F slit with embedded structure design, patch unit line width and pitch are $0.4 \mathrm{~mm}$, the unit side length of $13 \mathrm{~mm}, 90 \mathrm{~mm} * 75 \mathrm{~mm}$ size of the area, the number of units can accommodate 42 , the F-type crevice embedded structure of high impedance surface used in airborne telemetry antenna below the antenna simulation results shown in Figure 8, Figure 9:

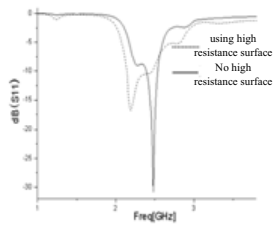

Figure 8. S11 curve

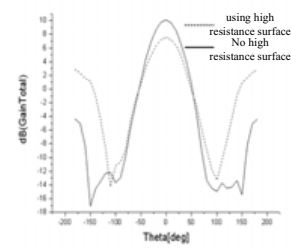

Figure 9. Antenna gain

The experimental results show that the gain of the aerial telemetry antenna is increased by $2.5 \mathrm{dBi}$ and the posterior lobe of the antenna is reduced by $7.4 \mathrm{~dB}$ after adding the high impedance surface of the F-type slot embedded structure. The high impedance surface is placed close to the antenna, The material is Rogers RO3210 (tm) with a thickness of $2 \mathrm{~mm}$, so the thickness of the antenna profile is 2 $\mathrm{mm}$ and the profile size is rather small compared to the quarter-wavelength profile of the metal reflector. The experimental results show that the bandwidth of the telemetry antenna is reduced in the process of reducing the thickness of the profile, and increasing the gain of the telemetry antenna by adding a new type of high resistance surface structure. Because the high-resistance surface structure is a resonant electromagnetic band-gap structure, and the in-phase reflection band is narrow. In addition, the reduction of the cell size leads to a further reduction of the band gap of the high resistance surface, since the coupling capacitance is increased by the change of the cell shape to realize the small size 
design but the inductance does not change. From the equation (1), as the coupling capacitance increases but the inductance does not change, the bandwidth of the high-resistance surface structure is reduced. Therefore, the size of the high-resistance surface structure is reduced and the bandwidth is also reduced during the miniaturization design.

\section{Bandgap design of high resistance surface}

In order to solve the problem of reducing the bandwidth while miniaturizing the design process by changing the shape of the cell, we first study the change of the reflected phase center frequency and the bandgap width from the different structural models of the high resistance surface. The mushroom-shaped patch structure, the butterfly-shaped patch-free structure, and the mushroom-shaped patch structure with short via were removed. We used different substrate materials to understand the change trend of the parameters of the high resistance surface, the purpose is to understand the relationship between the band gap width of the high resistance surface and the patch cycle.

As shown in Figure. 10, the horizontal axis represents the value of $a \varepsilon_{\mathrm{r}}^{1 / 2}$, and the vertical axis represents the value of the bandgap width.

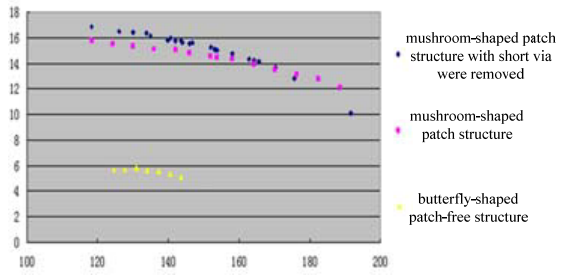

Figure 10. Bandwidth and patch cycle

It is found that the bandwidth of the same patch-like structure is the same curve with different substrate materials, but the patch shape of the butterfly shape is shorter than that of the mushroom-type structure, but the band width is smaller in the experiment, we found that the short-circuit via of the mushroom-type structure were removed, which still had the characteristic of high resistance surface structure, and the change of the center frequency and bandwidth of the reflection phase was not significant. The change of cell shape will not increase the band gap width.

The equivalent impedance and surface reflection coefficient of the high resistance surface structure are:

$$
\begin{gathered}
z=\frac{j \omega L}{1-\omega^{2} L C}=j X_{0} \\
\Gamma=\frac{Z-\eta}{Z+\eta}=\exp \left(j \varphi_{0}\right)
\end{gathered}
$$

from (3) and (4):

$$
\frac{d \varphi_{0}}{d \omega}=\frac{-2 \eta L\left(1+\omega^{2} L C\right)}{(\omega L)^{2}+\eta^{2}\left(1-\omega^{2} L C\right)^{2}}
$$

When $\omega=1 / \sqrt{\mathrm{LC}}$, the surface impedance of the high resistance surface is infinite. When the resonant frequency is fixed, increasing the thickness will increases the value of L, which means decreasing $\mathrm{C}$, which is beneficial to bandwidth broadening. 
The bandwidth of the high resistance surface refers to the frequency range corresponding to the phase of $\varphi_{0}= \pm 90^{\circ}$, so the formula (6) can be derived:

From (1), (2), (3), we can get

$$
\Delta \omega=\frac{1}{\eta C}=\frac{\omega_{0}^{2} L}{\eta}
$$

$$
\begin{aligned}
& \frac{B W}{f_{0}}=\frac{\Delta \omega}{\omega_{0}}=\frac{1}{2\left(\varepsilon_{1}+\varepsilon_{2}\right)} \frac{\lambda_{0}}{w} \frac{1}{\operatorname{acosh}(\mathrm{a} / g)} \\
& \frac{B W}{f_{0}}=\frac{\Delta \omega}{\omega_{0}}=2 \pi \mu_{r} \frac{t}{\lambda_{0}}
\end{aligned}
$$

In addition to the parameters of the chip structure, the dielectric constant of the substrate, the permeability of the substrate and the thickness of the substrate can be improved by using the substrate surface resistivity of the high resistance surface, from the formulas (7) and (8). Therefore, in order to improve the bandgap width, we can use a low dielectric constant substrate, high permeability substrate and to improve the substrate thickness to achieve. If the other parameters unchanged, the use of low dielectric constant substrate and improve the bandwidth at the same time, it will increase the resonant frequency of high resistance surface, which is not conducive to reducing cell size; high permeability substrate or substrate thickness can significantly increase the bandgap width, but also help to reduce the cell size. The high resistance surface structure of Figure. 2 is adopted to carry on the simulation, the simulation result is shown as in Figure.11, Figure.

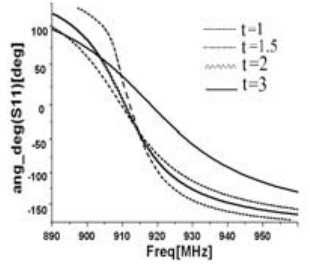

Figure 11. Thickness

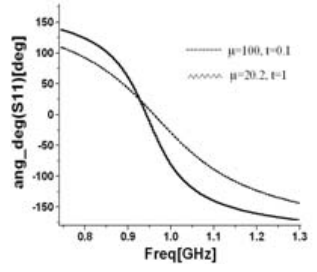

Figure 12. Magnetic

In order to observe the effect of increasing substrate thickness on improving the bandgap width, the patch period is kept constant and the inter-chip gap width is adjusted to maintain the resonant frequency. As shown in formula (7) and (8), the band gap width increases as the thickness of the substrate increases, and the increase is proportional to the result of Fig. Substrate thickness affects the magnitude of the equivalent inductance. As the thickness increases, the inductance increases and the resonant frequency decreases. It is feasible to increase the thickness of the substrate to increase the width of the bandgap compared with the height of the quarter-free-space wavelength of the metal reflector. It can be seen from Fig. 12 that the bandwidth of the substrate after using the high permeability material is obviously improved. Compared with the common substrate material of the same thickness, the band gap of the high permeability material substrate is remarkably improved, as shown in the formula (8) Large magnets and magnetic permeability is proportional to the size. Therefore, the permeability of the substrate increases, the substrate thickness can be very small, the patch size will be dramatically reduced, while also having a wide band gap width. Therefore, the use of high permeability material is to achieve high resistance to the surface structure of the ideal choice.

\section{Conclusion}

Based on the theoretical model of high resistance surface, a new type of slotted embedded structure is designed by analyzing the equivalent circuit model. Experimental results show that the new structure 
can greatly reduce the aerial telemetry antenna profile size while improving the antenna gain. In this paper, the equivalent circuit model of the high resistance surface structure is analyzed, and the parameters affecting the bandgap are pointed out. The method to increase the band gap is also found, and the contradiction between reducing the size and increasing the bandwidth is also solved. The structure and design ideas presented in this paper have practical significance for the design of modern airborne telemetry antenna.

\section{References}

1. P.F. Huo, H.H Li. Telemetry transmission quality and adjustment of RF system. measurement and control technology, 2008,27 (1): 4-6

2. Huang Wei, Xu Hengjie, Zhang Li. Electromagnetic simulation analysis of airborne multi antenna system. computer simulation, 2010,27 (5): 235-238

3. Yablonovitch E. Inhibited spontaneous emission in solid-state physics and electronics. Physical Review Letters. 1987, 58(20): 2059-2062

4. Wu Hao, Wang Lingmin, Tian L, et al. Design of microstrip antenna based on high impedance surface microwave photonic crystal . Journal of Jilin University (SCIENCE EDITION), 2014,52 (3): 583-586

5. Fu Yunqi, Yuan Naichang, Wen XISEN. Microwave photonic crystal antenna technology. Defense Industry Press.2006,24 (6)

6. M F Abedin, M Ali. Effects of a smaller unit cell planar EBG structure on the mutual coupling of a printed dipole array. IEEE Antennas and Wireless Propagation Let-ters, 2005, 4: 274-276.

7. Yan Dunbao. Doctoral dissertation of artificial magnetic conductor structure and application of. of national defense science and Technology University, 2006 